\title{
The genome sequence of the ethanologenic bacterium Zymomonas mobilis ZM4
}

\author{
Jeong-Sun Seo ${ }^{1,2,5}$, Hyonyong Chong ${ }^{1,3,5}$, Hyun Seok Park ${ }^{1,4}$, Kyoung-Oh Yoon ${ }^{1}$, Cholhee Jung ${ }^{1}$, \\ Jae Joon Kim ${ }^{1}$, Jin Han Hong ${ }^{1}$, Hyungtae Kim ${ }^{1}$, Jeong-Hyun Kim ${ }^{1}$, Joon-Il Kil ${ }^{1}$, Cheol Ju Park ${ }^{1}$, \\ Hyun-Myung $\mathrm{Oh}^{3}$, Jung-Soon Lee ${ }^{3}$, Su-Jung Jin ${ }^{3}$, Hye-Won $\mathrm{Um}^{3}$, Hee-Jong $\mathrm{Lee}^{3}$, Soo-Jin $\mathrm{Oh}^{3}$, \\ Jae Young Kim ${ }^{3}$, Hyung Lyun Kang ${ }^{3}$, Se Yong Lee ${ }^{1}$, Kye Joon Lee ${ }^{3}$ \& Hyen Sam Kang ${ }^{3}$
}

We report the complete genome sequence of Zymomonas mobilis ZM4 (ATCC31821), an ethanologenic microorganism of interest for the production of fuel ethanol. The genome consists of 2,056,416 base pairs forming a circular chromosome with 1,998 open reading frames (ORFs) and three ribosomal RNA transcription units. The genome lacks recognizable genes for 6-phosphofructokinase, an essential enzyme in the Embden-Meyerhof-Parnas pathway, and for two enzymes in the tricarboxylic acid cycle, the 2-oxoglutarate dehydrogenase complex and malate dehydrogenase, so glucose can be metabolized only by the Entner-Doudoroff pathway. Whole genome microarrays were used for genomic comparisons with the $Z$. mobilis type strain ZM1 (ATCC10988) revealing that 54 ORFs predicted to encode for transport and secretory proteins, transcriptional regulators and oxidoreductase in the ZM4 strain were absent from ZM1. Most of these ORFs were also found to be actively transcribed in association with ethanol production by ZM4.

Growing environmental concerns over the use and depletion of nonrenewable energy resources, together with the recent price increases and instabilities in the international oil markets have stimulated an increasing interest in the use of fermentation processes for the large-scale production of alternative fuels such as ethanol. As such, ethanol-producing microorganisms, such as the Gram-negative bacterium $Z$. mobilis, have potential for the production of fuel ethanol.

Z. mobilis, which is used in the tropics to produce pulque and alcoholic palm wines, uses the Entner-Doudoroff (ED) pathway to metabolize glucose, which results in only 1 mole of ATP being produced per mole of glucose $\mathrm{e}^{1}$. The potential advantages of using Z. mobilis for ethanol production include: (i) its high and specific rates of sugar uptake and ethanol production, (ii) its production of ethanol at yields close to the theoretical maximum with relatively low biomass formation, (iii) its high ethanol tolerance of up to $16 \%$ (vol/vol) and (iv) its facility for genetic manipulation ${ }^{2-6}$. However, wild strains of Z. mobilis can use only glucose, fructose and sucrose as carbon substrates, so recent research has focused on the development of recombinant strains capable of using pentose sugars ${ }^{7,8}$ for the conversion of cheaper lignocellulosic hydrolysates to ethanol. Improved mutants $^{9-11}$ as well as the application of metabolic flux analysis, sitedirected mutagenesis, specific gene deletion/insertion and metabolic engineering for strain developlment ${ }^{12,13}$ have also been reported. A physical map of $Z$. mobilis ZM4 genome and the ribosomal transcriptional unit have been previously reported ${ }^{14,15}$. In the current paper, the features of the complete sequence of the Z. mobilis ZM4 genome are presented and genomic characters are compared with those of another Z. mobilis strain, ZM1.

\section{RESULTS}

General features

The complete genome of $Z$. mobilis ZM4 consists of a single circular chromosome of 2,056,416 bp with an average G+C content of $46.33 \%$ (Table 1 and Supplementary Table 1 online). The 1,998 predicted coding ORFs cover $87 \%$ of the genome, and each ORF has an average length of $898 \mathrm{bp}$. Among these, 1,346 (67.4\%) could be assigned putative functions, 258 (12.9\%) were matched to conserved hypothetical coding sequences of unknown function and the remaining 394 (19.7\%) showed no similarities to known genes. The functions of the predicted ORFs were categorized by comparison with the COG database (Table 2).

Of the $0.84 \%$ of the genome that encodes stable RNA, 51 genes encode transfer RNAs, corresponding to 42 different isoacceptortRNA species. Of these ribosomal RNA transcriptional units, $r r n A$ is located at coordinate $140,000, r r n B$ at 360,000 and $r r n C$ at 520,000, all three being transcribed in the same predicted direction of replication.

The replication origin predicted by calculating GC skew $(\mathrm{G}-\mathrm{C} / \mathrm{G}+\mathrm{C})$ values $^{16}$ (Fig. 1) closely coincided with a 656-bp region containing one copy of a likely site (5'-GATCTNTTNTTTT- $\left.3^{\prime}\right)$ for initial DNA unwinding, and eight copies of probable sites

\footnotetext{
${ }^{1}$ Macrogen Inc., World Meridian Venture Center, 60-24, Gasan-dong, Seoul 153-781, Korea. 2Department of Biochemistry and Ilchun Molecular Medicine Institute, Medical Research Center, College of Medicine, Seoul National University, Seoul 110-799, Korea. ${ }^{3}$ Department of Microbiology, School of Biological Sciences, Seoul National University, Seoul 151-742, Korea. ${ }^{4}$ Department of Computer Science, Ewha Womans University, Seoul, 120-750, Korea. ${ }^{5}$ These authors contributed equally to this work. Correspondence should be addressed to J.-S.S. (jeongsun@macrogen.com) or H.S.K. (khslab@snu.ac.kr).
} 
Table 1 General features of the $Z$. mobilis genome

\begin{tabular}{ll}
\hline Length (bp) & $2,056,416$ \\
$\mathrm{G}+\mathrm{C}$ content (\%) & 46.33 \\
Open reading frames & \\
Coding region of genome (\%) & 87 \\
Total number of predicted ORFs & 1,998 \\
ORFs with assigned function & $1,346(67.4 \%)$ \\
Conserved hypothetical protein & $258(12.9 \%)$ \\
ORFs with no database match & $394(19.7 \%)$ \\
RNA element & \\
Stable RNA (percent of genome) & $0.84 \%$ \\
16S, 23S and 5S rRNA genes & 3 \\
tRNA & 51 \\
\hline
\end{tabular}

(5'-TTATNCANA- $\left.3^{\prime}\right)$ for DnaA binding. We also found that genes such as parA and parB, which are involved in chromosome partitioning, and gidA and $g i d B$, the glucose-inhibited division genes, were also located near the origin, which has often been observed in other bacterial genomes ${ }^{17}$.

\section{Comparison with other sequenced genomes}

Comparison of the $Z$. mobilis ZM4 ORFs (amino acid sequences) with those of other organisms revealed that 768 out of 1,668 ORFs listed in 을 the COG database have the closest similarity to the corresponding ORFs of Novosphingobium aromaticivorans (Supplementary Table 2 online) in line with a previous phylogenetic study on Z. mobilis ZM4 based on the $16 \mathrm{~S}$ ribosomal RNA sequence, where it was found that Z. mobilis ZM4 belonged to the Sphingomonas spp. group ${ }^{15}$. In particular, the ORFs classified into COG category J (translation, ribosomal structure and biogenesis) and category D (cell division and chromosome partitioning) showed high similarities to $N$. aromaticivorans. In contrast, only 2 out of 40 total ORFs classified into the COG category $\mathrm{N}$ (cell motility) and 5 out of 25 in category $\mathrm{V}$ (defense mechanisms) matched ORFs of N. aromaticivorans.

\section{General metabolism}

Z. mobilis uses glucose, fructose and sucrose anaerobically through the ED pathway, leading to the production of ethanol and $\mathrm{CO}_{2}$ (ref. 1). Analysis of the $Z$. mobilis genome sequence revealed the determinants of hexose-metabolizing enzymes such as invertase (ZMO0375, ZMO0942), levansucrase (ZMO0374), glucokinase (ZMO0369), glucose-6-phosphate isomerase (ZMO1212) and glucose-fructose oxidoreductase (ZMO0689) that would enable $Z$. mobilis to use sucrose, fructose and glucose as well as probably mannose, raffinose and sorbitol. However, there are no obvious genes for using lactose, maltose or cellobiose.

In the ED pathway, glucose-6-phosphate dehydrogenase (zwf, ZMO0367) oxidizes glucose-6-phosphate to 6-phosphonolactone. The lactone is dehydrated to 6-phosphogluconate by lactonase (ZMO1478). 6-phosphogluconate is dehydrated by 6-phosphogluconate dehydratase (edd, ZMO0368) to yield 2-keto-3-deoxy-6-phosphogluconate (KDPG). KDPG aldolase (eda, ZMO0997) cleaves KDPG to form pyruvate and glyceraldehyde-3-phosphate (Fig. 2). Glyceraldehyde-3phosphate is then metabolized via the triose phosphate common to the Embden-Meyerhof-Parnass (EMP) pathway to yield ethanol and carbon dioxide. All the genes for all of the enzymes of the EMP pathway except 6-phosphofructokinase are present in Z. mobilis (Fig. 2). The $z w f$ and edd genes are clustered with glf (ZMO0366; encodes facilitated diffusion protein for glucose) and glk (ZMO0369; glucokinase), whereas eda is separately located. This contrasts with
Table 2 Functional categories of predicted genes in Z. mobilis genome

\begin{tabular}{lr} 
COG categories & No. of gene \\
\hline Information storage and processing & \\
J. Translation, ribosomal structure and biogenesis & 141 \\
K. Transcription & 85 \\
L. DNA replication, recombination and repair & 87 \\
Cellular processes & \\
D. Cell cycle control, mitosis and meiosis & 20 \\
V. Defense mechanisms & 25 \\
T. Signal transduction mechanisms & 60 \\
M. Cell wall/membrane biogenesis & 120 \\
N. Cell motility & 40 \\
U. Intracellular trafficking and secretion & 47 \\
O. Post-translational modification, protein turnover, chaperones & 81 \\
Metabolism & \\
C. Energy production and conversion & 85 \\
G. Carbohydrate transport and metabolism & 76 \\
E. Amino acid transport and metabolism & 171 \\
F. Nucleotide transport and metabolism & 54 \\
H. Coenzyme transport and metabolism & 96 \\
I. Lipid transport and metabolism & 53 \\
P. Inorganic ion transport and metabolism & 93 \\
Q. Secondary metabolites biosynthesis, transport and catabolism & 32 \\
Poorly characterized & \\
R. General function prediction only & 198 \\
S. Function unknown & 104 \\
Not in COG & 539
\end{tabular}

All genes were classified according to the COG classification. http://www.ncbi.nlm.nih.gov/COG/

Escherichia coli, in which $z w f$, edd and eda are closely linked although regulation of the $z w f$ and edd-eda operon is independent ${ }^{17}$. By using the ED pathway instead of the EMP pathway, Z. mobilis yields only 1 mole of ATP per mole of fermented hexose, and produces ethanol at a theoretical yield of 2 moles/mole of substrate. Rapid production and high yield of ethanol as the only sugar fermentation product can be attributed to the presence of pyruvate decarboxylase (ZMO1360), an enzyme not frequently observed in bacteria, and two highly specific alcohol dehydrogenases (ZMO1236, ZMO1596).

The genes encoding two enzymes in the tricarboxylic acid cycle-the 2-oxoglutarate dehydrogenase complex and malate dehydrogenasewere not found. However, all the key building blocks, including oxaloacetate, malate, fumarate and succinate have been detected by means of high-performance liquid chromatography, and Z. mobilis is known to be able to synthesize all essential amino acids except for lysine and methionine. These results strongly indicate that other metabolic pathways are involved in producing oxaloacetate, malate, fumarate and succinate. Oxaloacetate can be produced from phosphoenolpyruvate and $\mathrm{CO}_{2}$ by phosphoenolpyruvate carboxylase (ZMO1496) or citrate lyase (ZMO0487: citrate $\leftrightarrow$ oxaloacetate + acetate). Malate can be synthesized by pyruvate carboxylation with malic enzyme (ZMO1955). Fumarate can be produced by fumarate dehydratase (ZMO1307). However, evidence for an alternative metabolic pathway for succinate production, such as the glyoxylate cycle, has not yet been found.

Although most genes for the pentose phosphate pathway are missing, all genes encoding enzymes necessary for the synthesis of phosphoribosyl-pyrophosphate, a precursor for purine/pyrimidine metabolism, are present. We also identified all genes required for the de novo biosynthesis of RNA and DNA. Z. mobilis possesses a complete set of genes for the sulfate reduction pathway as well as all 


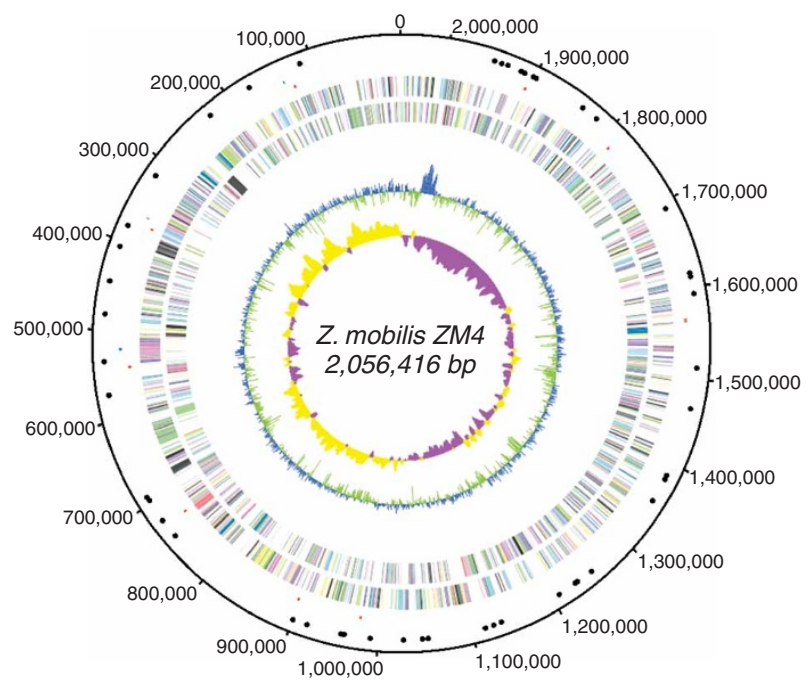

Figure 1 Overall features of the Z. mobilis ZM4 genome. The putative origin of replication is around $0 \mathrm{~kb}$. The outer scale indicates the coordinates in base pairs. The distribution of genes is shown on the first two rings within the scale according to the direction of the reading frame. The locations of rRNA and tRNA genes are shown by green dots and black dots, respectively. Putative transposases are shown by red dots. The next circle shows GC content values. Cyan and green colors indicate positive and negative signs, 윽 respectively. The central circle shows $\mathrm{GC}$-skew values $(\mathrm{G}-\mathrm{C} / \mathrm{G}+\mathrm{C})$ of the third bases of codons measured over the genome. Yellow and purple colors denote positive and negative signs, respectively. The window size was 10,000 nucleotides and the step size was 1,000 nucleotides.

the genes required for the synthesis of all amino acids, except for one gene in the lysine $(y f d Z)$ and one gene in the methionine (metB) pathways. For vitamins, all genes for riboflavin and folate synthesis and most genes for thiamin, ubiquinone, $\mathrm{NAD}^{+}$and pyridoxal are present. The absence of genes for pantothenate and biotin biosynthesis genes is in accordance with the known nutritional requirement of Z. mobilis for these vitamins.

\section{Transport systems and motility}

We recognized 180 genes encoding transport-related membrane proteins, on the basis of a search of the Transport Protein Database (http://tcdb.ucsd.edu/index.php). The largest number (83) of these proteins were electrochemical potential-driven transporters (class 2), and included 20 involved in iron metabolism, 13 multi-drug resistance exporters, three members of the resistance nodulation cell-division family, eight permeases of the major facilitator superfamily, seven cation transporters, seven amino acid transporters, three nucleoside permeases and four sugar transporters. There are several ORFs for the sec-independent protein secretion pathway and others for the TonBExbB-ExbD/TolA-TolQ-TolR (TonB) family of auxiliary proteins for energization of outer membrane receptor-mediated active transport systems. The second most numerous class (55) contained primary active transporters (class 3 ), including 41 members of the ATP-binding cassette $(A B C)$ transporter superfamily. There were five ORFs for the sec-dependent general secretory pathway, two for type III secretory pathway proteins and four for the type IV secretory pathway. The third largest class (14 members) was the channels/pores (class 1), consisting of five capsule polysaccharide export proteins and two carbohydrate (glucose)-facilitated diffusion proteins. The four remaining classes were group translocators (class 4; 4 ORFs), transport electron carriers (class 5; 3 ORFs), accessory factors involved in

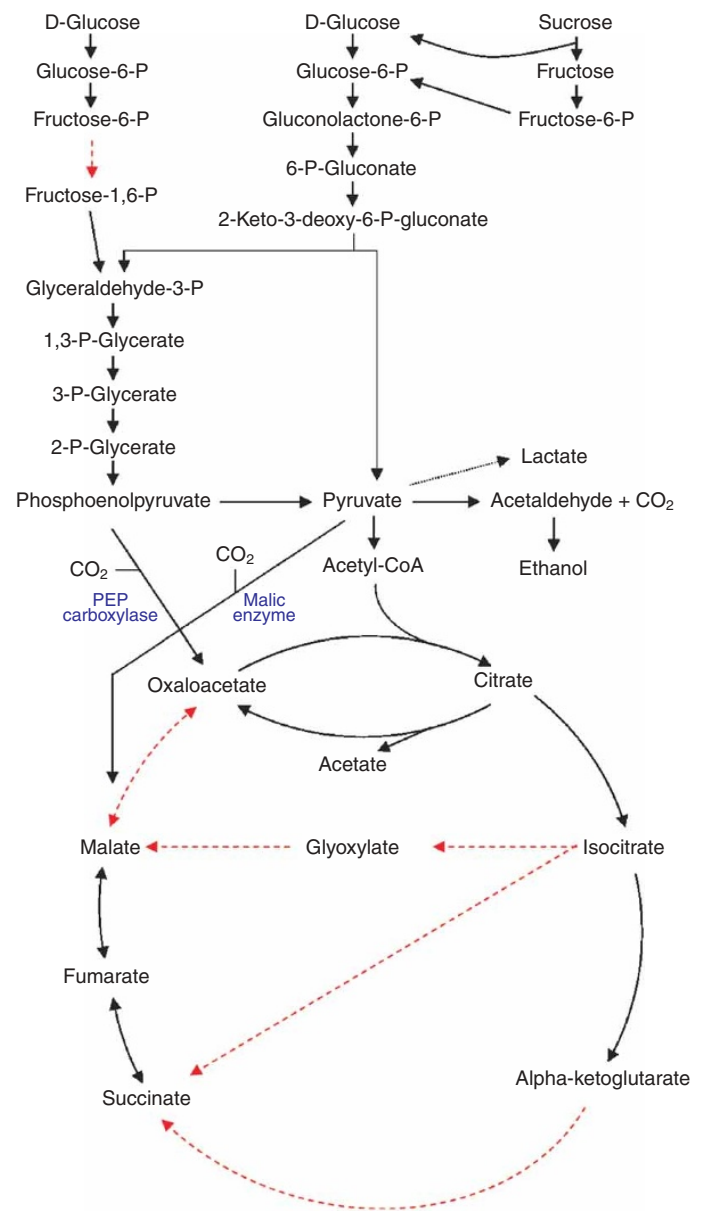

Figure 2 Central metabolic pathways of sugars. Enzymes missing from Z. mobilis are represented by red dotted arrows.

transport (class $8 ; 1 \mathrm{ORF}$ ) and incompletely characterized transport systems (class 9; 20 ORFs).

The flagellar cluster consists of 32 ORFs (ZMO0602-ZMO0652: flgABCDEFGHIJKL, flhAB, fliDEFGHIKLMNPQRS, motAB) encoding flagellar structure proteins, motor proteins and biosynthesis proteins. Classical chemotaxis signal transduction genes (cheABDRWY) and methyl-accepting chemotaxis genes ( $m c p A J)$, similar to those in E. coli, were present.

\section{Oxidative stress and respiration}

$Z$. mobilis is not an obligatory but a facultative anaerobe, implying that there must be a defense mechanism against oxidative stress. The most well-known reduction-oxidation cycling machinery is the glutathione system. Both glutathione reductase (ZMO1211) and glutathione synthase (ZMO1913) are present, as well as a Gamma-glutamylcysteine synthetase (ZMO1556). Genes encoding a catalase (ZMO0928), an iron-dependent superoxide dismutase (Fe-SOD; ZMO1060) and two kinds of peroxidases (ZMO1136, ZMO1573), which are thought to be responsible for protection from the toxic effects of superoxide and hydrogen peroxide in most aerobic organisms, are also present.

In addition to the genes that respond to oxidative stress, the genome contained several genes related to the electron transport system such as the Fe-S-cluster redox enzyme (ZMO1032), cytochrome $b$ (ZMO0957), cytochrome $c 1$ (ZMO0958), cytochrome 
c-type biogenesis proteins (ZMO1252-1256), electron transfer flavoprotein (ZMO1479, ZMO1480) and a ubiquinone biosynthesis protein (ZMO1189, ZMO1669). Genes for electron donor and receptor modules such as NADH dehydrogenase (ZMO1113) NADH: flavin oxidoreductase (ZMO1885), NADH:ubiquinone oxidoreductase complex (ZMO1809-1814), nitroreductase (ZMO0678) and fumarate reductase (ZMO0569) were also found. However, genes for cytochrome $o$ and cytochrome $d$, which use oxygen as a final electron acceptor, appeared to be absent.

It was reported that $Z$. mobilis has a respiratory electron transport chain ${ }^{19}$ and that it shows elevated molar growth yield during exponential aerobic growth ${ }^{20}$. Relative to anaerobic conditions, this leads to a decrease in the yield of ethanol and an accumulation of other less reduced metabolites such as acetaldehyde, acetone and acetate ${ }^{21,22}$. These results indicate that some NADH is oxidized in the respiratory chain with the simultaneous participation of the alcohol dehydrogenase reaction in aerobic culture conditions.

\section{Stress adaptation}

Protein denaturation and aggregation, resulting from exposure to heat or other stresses such as ethanol, are severe problems for cells, and are combated by induction of highly conserved heat shock proteins, whose function is to remove or refold the damaged cellular proteins ${ }^{23}$. Z. mobilis, an efficient ethanol producer, exhibits very high 을 ethanol tolerance ${ }^{3}$. The $Z$. mobilis contains ORFs for the complete sets of heat shock-responsive molecular chaperones, such as DnaK (ZMO0660), DnaJ (ZMO0661, ZMO1069, ZMO1545, ZMO1546, ZMO1690) and GrpE (ZMO0016) of the HSP-70 chaperone complex, GroES (ZMO1928; HSP-10), GroEL (ZMO1929; HSP-60) and HSP-33 (ZMO0410). ATP-dependent heat shock-responsive proteases, such as HslVU (ZMO0246, ZMO0247) and Clp (ZMO0948, ZMO0949, ZMO1424), were also found. As in the well-known E. coli system ${ }^{23}$, genes for alternative sigma factors, sigma-32 $\left(\sigma^{32}\right.$; ZMO0749) and sigma-E $\left(\sigma^{\mathrm{E}}\right.$; ZMO1404), for the pertinent responses against various stresses are present. It is known that sigma-32 of E. coli induces a 'classic' set of chaperones, proteases and other heat shock proteins, (2) thereby playing a central role in heat shock responses, whereas sigmaE induces periplasmic protease, chaperone and sigma-32 factor by

specific extracytoplasmic stress. It is also well known that the induction of sigma-32 factor is turned on when E. coli cells grown at $30{ }^{\circ} \mathrm{C}$ are shifted to $42{ }^{\circ} \mathrm{C}$, whereas proteins encoded by the sigma-E regulon are rapidly induced when $E$. coli cells are exposed to a more extreme temperature (e.g., $50{ }^{\circ} \mathrm{C}$ ) or $10 \%$ ethanol ${ }^{23}$. We suppose that sigma-E plays a key role in resisting high ethanol conditions in Z. mobilis. We also found genes for a sigma-E positive regulator (ZMO1842) and a transcriptional regulator of heat shock genes (ZMO0015), two tight regulators of heat shock gene expression.

The appropriate controls of gene expression are carried out by a combination of basic transcriptional machineries, including RNA polymerase and sigma factors. Genes for other sigma factors, $\sigma^{70}$ (rpoD; ZMO1623), $\sigma^{54}$ (rpoN; ZMO0274), and $\sigma^{28}$ (fliA; ZMO0626) were also found in the genome of $Z$. mobilis. We also identified 54 transcriptional activators and repressors.

\section{Higher G+C-content genes found only in strain ZM4}

To compare the $Z$. mobilis ZM4 genome with the unsequenced type strain (ZM1: ATCC10988) of Z. mobilis, labeled ZM1 and ZM4 genomic DNA were cohybridized with DNA microarrays containing probes for all the ORFs of $Z$. mobilis ZM4. It was found that most of the probes on the microarray hybridized equally with both labeled genomic DNAs (Fig. 3a). In addition, the two strains showed similar patterns of gene expression in microarray analysis of cultures grown under various growth stages (data not shown). Probably the overall genome structure of ZM1 and ZM4 is very similar.

However, it is interesting to note that strain ZM4 contains sequences that are absent from ZM1. These sequences consist of 54 genes that are clustered separately in five regions. Among the products of the 54 ORFs, there were four kinds of membrane transport proteins, and four kinds of proteins involved in a type IV secretory system, an oxidoreductase related to short chain alcohol dehydrogenase and several transcriptional regulators (Table 3). Two genes, $b c b G$ (ZMO1299) and $b c b E$ (ZMO1300), encoding capsular polysaccharide biosynthesis proteins, were also peculiar to strain ZM4. One of the five clusters, spanning from $1,984,100 \mathrm{nt}$ to $2,009,434 \mathrm{nt}(25.3 \mathrm{~kb})$, contains 25 ORFs and shows a higher G+C content (61.0\%) (Fig. 1) than the average $(46.3 \%)$ for the full genome of ZM4. The 25.3-kb sequence contains some interesting ORFs: ZMO1930 for phage-related integrase, ZMO1941 for conjugal transfer TraF protein, ZMO1954 conjugal transfer TrbL protein, and ZMO1933 and ZMO1934 for type I restriction-modification enzyme $S$ and $M$ subunits, respectively.

Most of the additional 54 ORFs in ZM4 were actively transcribed during the exponential growth phase, when ethanol is vigorously produced (Fig. $3 \mathbf{b}$ ). Global expression profiles of the ZM1 and ZM4 strains were analyzed in a sample taken when half of the glucose $(50 \mathrm{~g} / \mathrm{l})$ in the medium had been consumed and the data showed that a total of 294 ORFs were upregulated more than twofold in ZM4 a

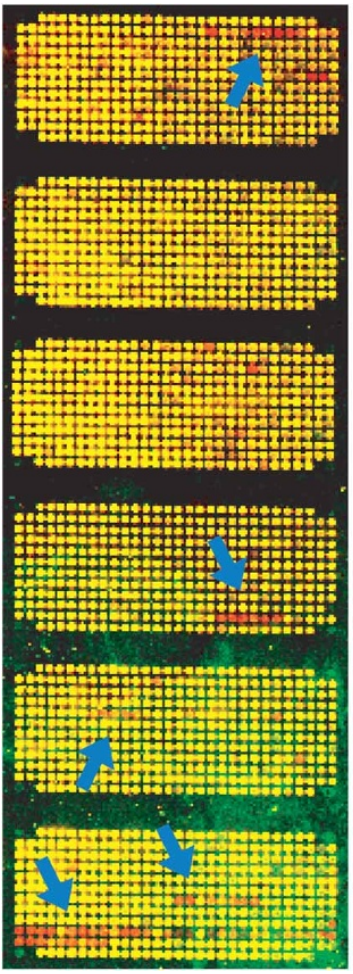

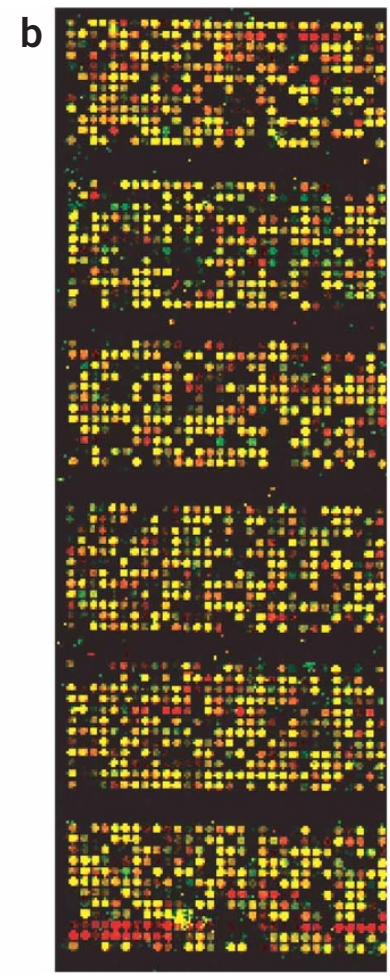

Figure 3 Comparison of genome structure and expression profiles between Z. mobilis ZM1 and ZM4. (a) Cohybridization of cy3-labeled ZM1 genomic DNA (green) and cy5-labeled ZM4 genomic DNA (red) on a Z. mobilis microarray. Arrows indicate extra sequences in strain ZM4.

(b) Cohybridization of cy3-labeled ZM1 cDNA (green) and cy5-labeled ZM4 cDNA (red) on a Z. mobilis microarray. Most ORFs in the extra sequence of strain ZM4 (same locations that arrows indicate on panel a) were actively expressed (red spot). RNAs were isolated at exponential growth phase. 
Table 3 Complete list of additional 54 ORFs in ZM4

\begin{tabular}{|c|c|c|c|}
\hline ZM00045 & hypothetical protein & ZM01863 & putative phosphatase \\
\hline ZM00046 & hypothetical protein & ZMO1864 & transposase \\
\hline ZM00047 & conserved hypothetical protein, transporter & ZMO1930 & phage-related integrase \\
\hline ZM00048 & hypothetical protein & ZM01931 & conserved hypothetical protein \\
\hline ZM00049 & hypothetical protein & ZM01932 & hypothetical protein \\
\hline ZM00050 & transcriptional regulator, LysR family & ZM01933 & type I restriction-modification enzyme, S subunit \\
\hline ZM00051 & hypothetical protein & ZM01934 & type I restriction-modification enzyme, M subunit \\
\hline ZM00052 & cyanate permease & ZM01935 & conserved hypothetical protein \\
\hline ZM00053 & beta-ketoadipate enol-lactone hydrolase, putative & ZM01936 & conserved hypothetical protein \\
\hline ZM00054 & transcriptional regulator, MarR family & ZM01937 & conserved hypothetical protein \\
\hline ZM00055 & permeases, predicted & ZM01938 & conserved hypothetical protein \\
\hline ZM01299 & capsular polysaccharide biosynthesis protein, bcbG & ZM01939 & ATPases involved in chromosome partitioning \\
\hline ZM01300 & capsular polysaccharide biosynthesis protein, bcbE & ZMO1940 & conserved hypothetical protein \\
\hline ZM01301 & conserved hypothetical protein & ZM01941 & type IV secretory pathway, conjugal transfer TraF transmembrane protein \\
\hline ZM01302 & lipoate-protein ligase B & ZMO1942 & type IV secretory pathway, VirD2 components (relaxase) \\
\hline ZM01459 & transporter, putative & ZM01943 & type IV secretory pathway, VirD2 components (relaxase) \\
\hline ZM01460 & thiosulfate sulfurtransferase (rhodanese) family protein & ZMO1944 & transcriptional regulatory protein \\
\hline ZM01461 & conserved hypothetical protein & ZMO1945 & predicted epimerase, $\mathrm{PhzC} / \mathrm{PhzF}$ homolog \\
\hline ZM01462 & conserved hypothetical protein & ZM01946 & oxidoreductase (short-chain alcohol dehydrogenases) \\
\hline ZM01463 & TonB-dependent receptor, probable & ZMO1947 & translational inhibitor protein \\
\hline ZM01856 & putative transport protein & ZMO1948 & conserved hypothetical protein \\
\hline ZM01857 & transcriptional regulator, probable & ZM01949 & $\mathrm{NAD}(\mathrm{P}) \mathrm{H}$ quinone oxidoreductase, putative \\
\hline ZM01858 & hypothetical protein & ZMO1950 & aspartate/tyrosine/aromatic aminotransferase \\
\hline ZM01859 & regulator of pathogenicity factors, carbohydrate-selective porin & ZM01951 & demethylmenaquinone methyltransferase \\
\hline ZMO1860 & similar to nodulin 21 & ZM01952 & 3-methyl-2-oxobutanoate hydroxymethyltransferase; PanB, probable \\
\hline ZM01861 & dioxygenases related to 2-nitropropane dioxygenase & ZMO1953 & hypothetical protein \\
\hline ZM01862 & hypothetical protein & ZMO1954 & type IV secretory pathway, VirB10, conjugal transfer TrbL transmembrane \\
\hline
\end{tabular}

compared to ZM1, whereas 153 ORFs were expressed more than twice in ZM1 (Supplementary Tables 3 and 4 online).

It has been reported that strain ZM4 is more tolerant of higher alcohol concentration than the type strain ZM1 and that ZM4 shows higher specific rates for growth, ethanol production and glucose ᄂ uptake ${ }^{5,24}$. Perhaps some of the genes peculiar to ZM4 and actively expressed at the higher glucose concentration will prove to be good

(2) target genes for constructing recombinant strains that ferment ethanol with higher productivity.

\section{(1)}

\section{DISCUSSION}

Analysis of the complete sequence of the Z. mobilis ZM4 genome reveals why this is one of the most powerful ethanol-producing microbes described, and suggests potential means to improve the yield and rate of ethanol production. Because $Z$. mobilis produces only one mole of ATP per mol of glucose via the ED pathway, Z. mobilis requires almost twice as much glucose as microbes that use the EMP pathway to produce equivalent amounts of ATP. The higher rate for glucose utilization and ethanol production are also supported by the fact that pyruvate decarboxylase and alcohol dehydrogenases are very highly expressed in $Z$. mobilis.

The absence of 6-phosphoglucokinase and the consequent dependence of $Z$. mobilis on the ED pathway raises interesting questions about the evolution of carbohydrate metabolism. The ED pathway is active in most Gram-negative bacteria and many other microorganisms including some archeabacteria ${ }^{25}$. The ubiquity of the ED pathway suggests that it is of far greater importance in nature than was previously recognized and indeed an essay on the evolution of glycolytic pathways suggested that the ED pathway predates the EMP pathway ${ }^{26}$. Although it is also possible that $Z$. mobilis is not able to use the EMP pathway as a result of the loss of the gene encoding 6-phosphoglucokinase, considering the genome size and relatively simple metabolic pathways present in Z. mobilis, it is more likely that the EMP pathway present in other microorganisms is the result of acquiring the 6-phosphoglucokinase gene.

The absence of two genes for the tricarboxylic acid cycle, the 2-oxoglutarate dehydrogenase complex and malate dehydrogenase, suggests the existence of alternative pathways to the tricarboxylic acid cycle. Because essential metabolites for cell growth are provided from the tricarboxylic acid cycle, this provides an explanation for the low biomass formation of $Z$. mobilis compared with other microorganisms in which the tricarboxylic acid cycle is actively operating 5 .

The observation that $Z$. mobilis ZM4 contains extra DNA sequences encoding for a total of 54 ORFs, compared to the genome of the type strain ZM1, raises questions about the origin as well as the role of these ORFs. Given that 25 ORFs in these high G+C-content DNA sequences show very high identity with some genes found in phages, and that there is little sequence homology with genes from other bacteria, the possibility exists that the higher $\mathrm{G}+\mathrm{C}$ content of the additional DNA sequences may have been horizontally transferred from phages. Plasmid exchange is another possible route, because the 3 -kb sequence in the additional DNA sequence exhibits substantial homologous regions with the sequence of Ralstonia solanacearum that encodes conjugal proteins TraF and TraL. Transposon-mediated gene transfer is also a possibility considering that the sequences encoding TraF and TraL are also homologous with Ralstonia oxalatica transposon $\operatorname{Tn} 4371$.

Among the 54 predicted ORFs, four ORFs that encode transport proteins or permeases, and two genes for $\mathrm{NAD}(\mathrm{P}) \mathrm{H}$ :quinone oxidoreductase (ZMO1949) and oxidoreductase (short-chain alcohol dehydrogenases; ZMO1946) were found to be very highly expressed. It is quite likely that these genes contribute to the higher rates of glucose uptake and ethanol production in the ZM4 strain. Two genes encoding capsular carbohydrate synthesis enzymes were also found to be actively 
expressed in the ZM4, and it is possible that they may contribute to resistance against osmotic pressure at the high concentration of glucose media and ethanol produced during fermentation. Thus, it is plausible that several of the characteristics of ZM4 that make it attractive as an ethanol producer may be attributable to DNA acquired comparatively recently.

\section{METHODS}

Sequencing and assembly. Genomic DNA from Z. mobilis ZM4 strain ATCC 31821 was sequenced using whole genome random shotgun methods ${ }^{27}$. Mechanically sheared 2-kb and 10-kb DNA fragments were isolated, inserted into pUC18 and cloned. Template preparation reactions were done using standard protocols. DNA sequencing reactions were carried out using PE BigDye Terminator chemistry, and sequencing ladders were analyzed on PE 3700 automated DNA sequencers. Approximately 40,000 reads with PHRED scores_20 were generated, providing a 14-fold genome coverage. These sequences were assembled by using the PHRED_PHRAP_CONSED software package $^{28}$ (http://www.phrap.org/). Both ends of 292 fosmid clones with an average insert size of $40 \mathrm{~kb}$ were also sequenced, providing a validation check of the final assembly. Sequencing gaps were closed by primer walking on gap-spanning clones and combinatorial PCR-assisted contig extension ${ }^{29}$.

Genome annotation. ORFs were predicted with the Glimmer software ${ }^{30}$, and functional annotation of predicted ORFs was carried out by an alignment search tool (Blastx) with a nonreviewed set database. Further analyses such as Pfam and COG (Clusters of Orthologous Genes) ${ }^{31}$ were carried out to find 윽 homologous protein domains and compare protein sequences between species.

Designing and spotting of oligonucleotides for microarrays. We designed 50-mer oligonucleotide probes representing each $Z$. mobilis ORF, as follows: melting temperatures were normalized within $2{ }^{\circ} \mathrm{C}$; the $\mathrm{G}+\mathrm{C}$ content of designed oligonucleotide probes was restricted to $46 \pm 2 \%$ matching the $46.33 \% \mathrm{G}+\mathrm{C}$ content of $Z$. mobilis; 'no sequence homology' to other regions of the genome was restricted to a maximum of $35 \mathrm{bp}$, with no exact sequence matches of more than $15 \mathrm{bp}^{32}$. The 2,112 oligonucloetide probes and 48 control probes, whose concentrations were normalized to $50 \mu \mathrm{M}$ (pmole/ $/ \mu \mathrm{l}$ ) in $50 \%$ DMSO, were spotted on CMT-GAP aminosilane-coated glass slides according to the order of the ORFs in the genome.

Labeling of genomic DNA and RNA. Genomic DNAs isolated from Z. mobilis (2) strains ZM1 and ZM4 were fluorescently labeled with random hexamers and either Cy3-labeled dCTPs or, Cy5-labeled dCTPs respectively, using the Klenow fragment of DNA polymerase. Total RNA was extracted using an RNeasy kit (Qiagen) with the RNA stabilizing solution, RNAlater (Ambion). We labeled $50 \mu \mathrm{g}$ of total RNA from strain ZM1 with Cy3-labeled dCTPs, and $50 \mu \mathrm{g}$ of total RNA from strain ZM4 was labeled with Cy5-labeled dCTPs, using reverse transcriptase (Superscript II; Invitrogen) with random hexamers ${ }^{33}$.

Nucleotide sequence accession number. The sequence reported in this paper has been deposited in GenBank with accession number AE008692.

Microarray data. Raw data files of microarray experiment are available at http://www.macrogen.com/zymomonas/microarray and EBI ArrayExpress DB with accession number E-MEXP-217.

Note: Supplementary information is available on the Nature Biotechnology website.

\section{ACKNOWLEDGMENTS}

We are very grateful to Peter L. Rogers and Keith Chater for thoughtful advice and discussions throughout the experimentation and manuscript preparation. H.S.K. would especially like to thank President Y.S. Song, for lending the automatic DNA sequencer Licor. This work was supported by an IMT-2000 grant from the Korean Ministry of Information and Communication and the Korean Ministry of Commerce, Industry and Energy [00016103]. J.S.L., S.J.J., H.W.U., H.J.L., S.J.O. and J.Y.K. were supported by a BK21 Research Fellowship from the Korean Ministry of Education and Human Resources Development.

\section{COMPETING INTERESTS STATEMENT}

The authors declare that they have no competing financial interests.
Received 28 June; accepted 6 October 2004

Published online at http://www.nature.com/naturebiotechnology/

1. Swings, J. \& De Ley, J. The biology of Zymomonas. Bacteriol. Rev. 41, 1-46 (1977).

2. Rogers, P.L., Lee, K.J. \& Tribe, D.E. Kinetics of alcohol production by Zymomonas mobilis at high sugar concentrations. Biotechnol. Lett. 1, 165-170 (1979).

3. Lee, K.J., Tribe, D.E. \& Rogers, P.L. Ethanol production by Zymomonas mobilis in continuous culture at high glucose concentration. Biotechnol. Lett. 1, 421-426 (1979).

4. Lee, K.J., Lefebvre, M., Tribe, D.E. \& Rogers, P.L. High productivity ethanol fermentations with Zymomonas mobilis using continuous cell recycle. Biotechnol. Lett. 2, 487492 (1980).

5. Lee, K.J., Skotnicki, M.L., Tribe, D.E. \& Rogers, P.L. Kinetic studies on a highly productive strain of Zymomonas mobilis. Biotechnol. Lett. 2, 339-344 (1980).

6. Rogers, P.L., Lee, K.J., Skotnicki, M.L. \& Tribe, D.E. Ethanol production by Zymomonas mobilis. Adv. Biochem. Eng 23, 37-84 (1982).

7. Zhang, M., Eddy, C., Deanda, K., Finkelstein, M. \& Picataggio, S. Metabolic engineering of a pentose metabolism pathway in ethanologenic Zymomonas mobilis. Science 267, 240-243 (1995).

8. Deanda, K., Zhang, M., Eddy, C. \& Picataggio, S. Development of an arabinosefermenting Zymomonas mobilis strain by metabolic pathway engineering. Appl. Environ. Microbiol. 62, 4465-4470 (1996).

9. Zaldivar, J., Nielsen, J. \& Olsson, J. Fuel ethanol production from lignocellulose: a challenge for metabolic engineering and process integration. Appl. Microbiol. Biotechnol. 56, 17-34 (2001).

10. Ingram, L.O. et al. Enteric bacterial catalysts for fuel ethanol production. Biotechnol. Prog. 15, 855-866 (1999).

11. Ingram, L.O. et al. Metabolic engineering of bacteria for ethanol production. Biotechnol. Bioeng. 58, 204-214 (1998).

12. Hatzimanikatis, V., Emmerling, M., Sauer, U. \& Bailey, J.E. Application of mathematical tools for metabolic design of microbial ethanol production. Biotechnol. Bioeng. 58, 154-161 (1998)

13. Cornish-Bowden, A. \& Cardenas, M.L. From genome to cellular phenotype-a role for metabolic flux analysis? Nat. Biotechnol. 18, 267-268 (2000).

14. Kang, H.L. \& Kang, H.S. A physical map of the genome of ethanol fermentative bacterium Zymomonas mobilis ZM4 and localization of genes on the map. Gene 206, 223-228 (1998)

15. Lee, J.S., Jin, S.J. \& Kang, H.S. Molecular organization of the ribosomal RNA transcription unit and the phylogenetic study of Zymomonas mobilis ZM4. Mol. Cells 11, 68-74 (2001).

16. Lobry, J.R. Asymmetric substitution patterns in the two DNA strands of bacteria. Mol. Biol. Evol. 13, 660-665 (1996).

17. Lewin, B. Creating the replication forks at an origin. in Genes $V$ (ed. Lewin, B.) 594-597 (Oxford Press, New York, 1994).

18. Moat, A.G., Foster, J.W. \& Spector, M.P. Central pathways of carbohydrate metabolism. in Microbial physiology (eds. Moat, A.G., Foster, J.W. \& Spector, M.P.) 351-367, 373-377 (Wiley-Liss, New York, 2002).

19. Strohdeicher, M., Neuss, B., Bringeer-Meyer, S. \& Sahm, H. Electron transport chain of Zymomonas mobilis. Interaction with the membrane-bound glucose dehydrogenase and identification of ubiquinone 10. Arch. Microbiol. 154, 536-543 (1990).

20. Zikmanis, P., Kruce, R. \& Auzina, L. An elevation of the molar growth yield of Zymomonas mobilis during aerobic exponential growth. Arch. Microbiol. 167, 167171 (1997).

21. Viikari, L. Carbohydrate metabolism in Zymomonas. Crit. Rev. Biotechnol. 7, 237-261 (1988).

22. Kalnenieks, U., Galinina, N., Toma, M.M. \& Poole, R.K. Cyanide inhibits respiration yet stimulates aerobic growth of Zymomonas mobilis. Microbiology 146, 1259-1266 (2000).

23. Yura, T. Regulation of heat shock response in bacteria. Annu. Rev. Microbiol. 47, 321-350 (1993).

24. Skotnicki, M.L., Lee, K.J., Tribe, D.E. \& Rogers, P.L. Genetic alteration of Zymomonas mobilis for ethanol production. Basic Life Sci. 19, 271-290 (1982).

25. Conway, T. The Entner-Doudoroff pathway: history, physiology, and molecular biology. FEMS Microbiol. Rev. 9, 1-27 (1992).

26. Romano, A.H. \& Conway, T. Evolution of carbohydrate metabolic pathways. Res. Microbiol. 147, 448-455 (1996).

27. Fleischmann, R.D. et al. Whole-genome random sequencing and assembly of Haemophilus influenzae Rd. Science 269, 496-512 (1995).

28. Gordon, D., Abajian, C. \& Green, P. Consed: a graphical tool for sequence finishing. Genome Res. 8, 195-202 (1998).

29. Tettelin, H., Radune, D., Kasif, S., Khouri, H. \& Salzberg, S.L. Optimized multiplex PCR: efficiently closing a whole-genome shotgun sequencing project. Genomics 62, 500-507 (1999).

30. Delcher, A.L. et al. Improved microbial gene identification with GLIMMER. Nucleic Acids Res. 27, 4636-4641 (1999).

31. Tatusov, R.L., Galperin, M.Y., Natale, D.A. \& Koonin, E.V. The COG database: a tool for genome-scale analysis of protein functions and evolution. Nucleic Acids Res. 28, 33-36 (2000).

32. Kane, M.D. et al. Assessment of the sensitivity and specificity of oligonucleotide (50 mer) microarrays. Nucleic Acids Res. 28, 4552-4557 (2000).

33. Richmond, C.S., Glasner, J.D., Mau, R., Jin, H. \& Blattner, F.R. Genome-wide expression profiling in Escherichia coli K-12. Nucleic Acids Res. 27, 3821-3835 (1999). 